\title{
Relationships between craniocervical posture and pain-related disability in patients with cervico-craniofacial pain
}

This article was published in the following Dove Press journal:

Journal of Pain Research

30 July 2015

Number of times this article has been viewed

\author{
Ibai López-de-Uralde- \\ Villanueva ${ }^{1-4}$ \\ Hector Beltran-Alacreu ${ }^{1-3}$ \\ Alba Paris-Alemany ${ }^{1-4}$ \\ Santiago Angulo-Díaz- \\ Parreño $2,3,5$ \\ Roy La Touche ${ }^{1-4}$
}

'Department of Physiotherapy, Faculty of Health Science, ${ }^{2}$ Research Group on Movement and Behavioral Science and Study of Pain, The Center for Advanced Studies University La Salle, Universidad Autónoma de Madrid, Aravaca, Madrid, Spain; ${ }^{3}$ Institute of Neuroscience and Craniofacial Pain (INDCRAN), Madrid, Spain; ${ }^{4}$ Hospital La Paz Institute for Health Research, IdiPAZ, Madrid, Spain; ${ }^{5}$ Faculty of Medicine, Universidad San Pablo CEU, Madrid, Spain
Correspondence: Roy La Touche Facultad de Ciencias de la Salud, Centro Superior de Estudios Universitarios La Salle, Calle La Salle, 10, 28023 Madrid, Spain

Tel +34917401980 ext 256

Email roylatouche@yahoo.es
Objectives: This cross-sectional correlation study explored the relationships between craniocervical posture and pain-related disability in patients with chronic cervico-craniofacial pain (CCFP). Moreover, we investigated the test-retest intrarater reliability of two craniocervical posture measurements: head posture (HP) and the sternomental distance (SMD).

Methods: Fifty-three asymptomatic subjects and 60 CCFP patients were recruited. One rater measured HP and the SMD using a cervical range of motion device and a digital caliper, respectively. The Spanish versions of the neck disability index and the craniofacial pain and disability inventory were used to assess pain-related disability (neck disability and craniofacial disability, respectively).

Results: We found no statistically significant correlations between craniocervical posture and pain-related disability variables (HP and neck disability $[r=0.105 ; P>0.05]$; HP and craniofacial disability $[r=0.132 ; P>0.05]$; SMD and neck disability $[r=0.126 ; P>0.05]$; SMD and craniofacial disability $[r=0.195 ; P>0.05])$. A moderate positive correlation was observed between HP and SMD for both groups (asymptomatic subjects, $r=0.447$; CCFP patients, $r=0.52$ ). Neck disability was strongly positively correlated with craniofacial disability $(r=0.79 ; P<0.001)$. The test-retest intrarater reliability of the HP measurement was high for asymptomatic subjects and CCFP patients (intraclass correlation coefficients $=0.93$ and 0.81 , respectively) and for SMD (intraclass correlation coefficient range between 0.76 and 0.99 ); the test-retest intrarater reliability remained high when evaluated 9 days later. The HP standard error of measurement range was $0.54-0.75 \mathrm{~cm}$, and the minimal detectable change was $1.27-1.74 \mathrm{~cm}$. The SMD standard error of measurement was $2.75-6.24 \mathrm{~mm}$, and the minimal detectable change was $6.42-14.55 \mathrm{~mm}$. Independent $t$-tests showed statistically significant differences between the asymptomatic individuals and CCFP patients for measures of craniocervical posture, but these differences were very small (mean difference $=1.44 \mathrm{~cm}$ for HP; $6.24 \mathrm{~mm}$ for SMD). The effect sizes reached by these values were estimated to be small for SMD $(d=0.38)$ and medium for HP $(d=0.76)$.

Conclusion: The results showed no statistically significant correlations between craniocervical posture and variables of pain-related disability, but a strong correlation between the two variables of disability was found. Our findings suggest that small differences between CCFP patients and asymptomatic subjects exist with respect to the two measurements used to assess craniocervical posture (HP and SMD), and these measures demonstrated high test-retest intrarater reliability for both CCFP patients and asymptomatic subjects.

Keywords: measurement, neck pain, rehabilitation, reliability, reproducibility of results, temporomandibular disorders

\section{Introduction}

In patients with head and neck pain, it is generally recommended that head posture (HP) be quantified as part of the exam process to facilitate the diagnosis and determine 
treatment strategies. In addition, it is important to monitor a patient's progress. ${ }^{1}$ A recent systematic review identified the presence of changes in the craniocervical posture in patients with temporomandibular disorders (TMD) of muscular origin compared with healthy controls; ${ }^{2}$ however, in another systematic review, conflicting conclusions on this subject were reached. ${ }^{3}$ Both reviews agree that the studies that were analyzed exhibited methodological flaws. ${ }^{2,3}$ Numerous studies have investigated the association between craniocervical posture and pain intensity, also with conflicting results. Some studies have reported differences in the HP of patients with neck pain compared with that of asymptomatic subjects, ${ }^{4}$ whereas other studies failed to detect such differences. ${ }^{5}$

Association studies between neck-pain-related disability and craniocervical posture have yielded controversial results; ${ }^{6-8}$ thus, it is difficult to draw clear conclusions at this time. There is also insufficient evidence to establish a relationship between craniofacial disability in patients with neck pain and TMD of muscular origin (cervico-craniofacial pain [CCFP]).

The growing interest in the importance of HP by researchers and clinicians alike is due to the belief that a forward HP (FHP) is associated with the development and persistence of certain disorders, such as cervical headaches and migraines, ${ }^{9}$ myofascial pain syndrome, ${ }^{10}$ and craniofacial pain in women. ${ }^{11}$ FHP is a common postural abnormality, ${ }^{12}$ in which the neck and the head rest in front of the shoulder (anterior to the vertical line of the body's center of gravity). Attempts have been made to quantify FHP using subjective methods ${ }^{13}$ as well as objective methods, such as photographs, ${ }^{14}$ radiographic images, ${ }^{15}$ or the cervical range of motion (CROM) instrument. ${ }^{16}$

The CROM device was designed to measure the CROM, but it can also measure protraction and retraction of the head. ${ }^{16}$ In the trial conducted by Garrett et al, ${ }^{16} \mathrm{HP}$ was measured in a sitting position using the CROM device. Another relevant measurement is the sternomental distance (SMD). This measurement is defined as the straight distance between the upper border of the manubrium sternum and the protuberance of the chin, and is considered a plausible objective indicator of head and neck mobility. ${ }^{17}$ We hypothesized a direct association between SMD and HP. However, we found only two studies that evaluated the SMD, and they did not associate it with HP. ${ }^{18,19}$ Furthermore, there is also little evidence concerning the reliability of intra- and interexaminer measurement of HP using a CROM device.

One of the goals of this study was to examine the intraand interexaminer reliability of using a digital caliper to measure the SMD. In addition, we assessed a possible association between HP and SMD measured using these instruments, and as an ultimate objective, we determined whether there are differences in craniocervical posture between asymptomatic subjects and patients with chronic CCFP.

The objective of this cross-sectional correlation study was to explore the relationships between craniocervical posture and pain-related disability in patients with chronic CCFP. Moreover, the reliability of the two craniocervical posture measurements (HP and the SMD) was investigated. The third objective was to compare the results of the craniocervical posture among asymptomatic subjects and patients.

\section{Methods \\ Study design}

This research employed a cross-sectional correlation design with a nonprobabilistic sample. In the second part of the study, we employed two-group repeated measures for intrarater reliability design (Figure 1). This study was planned and conducted in accordance with the "Strengthening the Reporting of Observational Studies in Epidemiology" (STROBE statement) ${ }^{20}$ and the "Guidelines for Reporting Reliability and Agreement Studies" (GRRAS). ${ }^{21}$

\section{Subjects}

Two convenience samples of asymptomatic subjects were obtained from our university campus and the local community using flyers, posters, and social media. The inclusion criteria were being between 18 and 65 years of age and having no neck or face pain during the data-collection period, and no a history of neck or face pain in the 6 months prior to the study.

The second convenience sample of symptomatic subjects consisted of chronic CCFP patients recruited from two private clinics specializing in spine, craniofacial pain, and TMD in Madrid, Spain. A diagnosis of CCFP of muscular origin was the first inclusion criterion. We defined CCFP of muscular origin as the presence of mechanical signs of dysfunction and muscular pain (eg, limited movement, uncoordinated movement, and weakness and lack of endurance in the neck and jaw) that were exacerbated by maintained postures and movement and that generated pain in the cervical and craniofacial regions. ${ }^{22}$ The additional inclusion criteria were: 1 ) signs of disability and pain in the orofacial and craniomandibular region according to the Craniofacial Pain and Disability Inventory (CF-PDI);23 2) a primary diagnosis of myofascial pain following Axis 1 (myofascial pain) of the Research Diagnostic Criteria for Temporomandibular Disorders; ${ }^{24}$ 


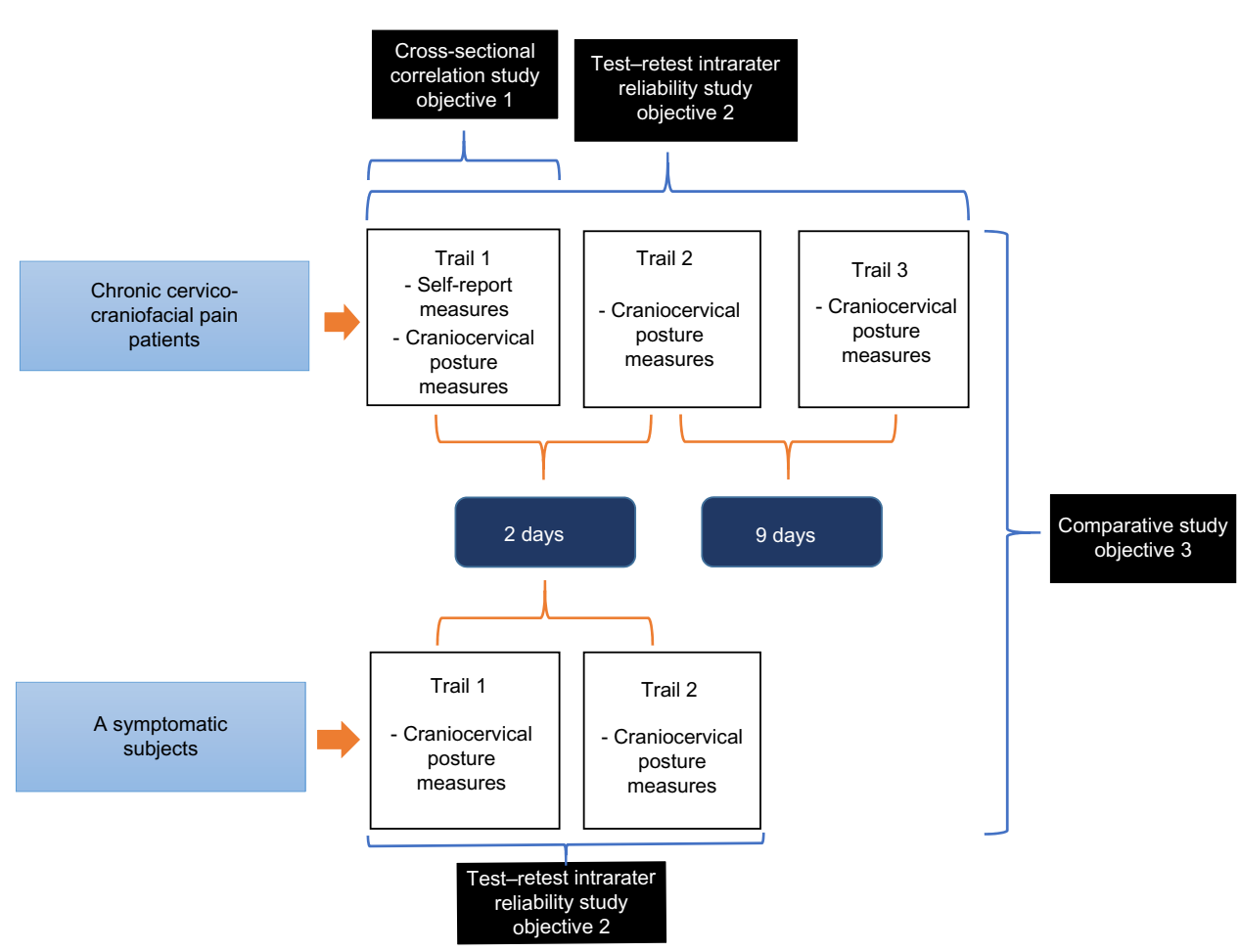

Figure I Flowchart of the study.

3) pain persisting for at least 6 months; 4) a neck disability index (NDI) score of at least $5 ;{ }^{25}$ and 5) bilateral pain of the temporal, masseter, suboccipital, and trapezius muscles. Patients were excluded if they had any of the following "red flags": ${ }^{26}$ a rheumatologic disease, any type of cancer, cervical radiculopathy, myelopathy, or a history of cervical surgery or whiplash trauma. The study was conducted in accordance with the Declaration of Helsinki and was approved by the local ethics committee. Prior to their participation, the subjects provided written informed consent.

\section{Evaluators}

The assessments were made by one physiotherapist with more than 3 years of clinical experience using the CROM device to measure range of movement and HP in clinical practice. The physiotherapist received a 120-minute training session on how to use the digital calipers and how to measure the SMD. The evaluator was blinded as to which participants were asymptomatic subjects versus patients.

\section{Instrumentation}

We used the CROM 3 (Performance Attainment Associates, Roseville, MN, USA) to measure HP. This device resembles eyeglasses and is made from lightweight plastic with three inclinometers, one for each plane of motion. It is adjusted using a hook-and-loop strap. The part of the device used to measure HP includes the forward head arm and the vertebra locator. The forward head arm is equipped with a ruler marked in $0.5 \mathrm{~cm}$ increments, indicating the horizontal distance between the bridge of the nose and the vertebra locator. The vertebra locator has a leveler bubble on top to assist the physiotherapist with accurate positioning.

To measure the SMD directly, we used a plastic digital caliper with a five-digit LCD display $\left(\right.$ Perel $^{\circledR}$; Velleman NV Instruments, Gavere, Belgium) that was able to measure in inches or millimeters ( $\mathrm{mm}$ ) and had a range of $0.01-150 \mathrm{~mm}$. It also includes a ruler provided with a nonius, for accurate measurement of lengths or angles. The one used for measuring length comprised a rule divided into equal parts on which a nonius slides such that $n-1$ divisions of the rule are divided into $n$ equal parts of the nonius. It has two tips for controlling internal and external measurements.

\section{Procedures}

The assessments were made between May 2012 and September 2014 in our university laboratory for the asymptomatic subjects, and between June 2014 and September 2014 for the CCFP patients. Each healthy subject visited the laboratory on two different occasions separated by 48 hours. Each patient visited the laboratory on three different occasions separated by 48 hours (between trials 1 and 2) and 9 days (between trials 2 and 3) (Figure 1). In addition, 
the participants who took any type of medication and/or received physical therapy, as well as those had any exclusion criteria during the time period between trials were counted as lost. For the CCFP patients, the assessment was always performed by the same rater. For both the healthy controls and the CCFP patients, the rater used a data collection sheet to record the measurements. Before the assessments, the subjects removed their eyeglasses, hats, and all jewelry. The measurements in this study were taken twice; the order in which they were performed was as follows.

\section{HP}

To quantify HP, the subjects were instructed to remain in the following starting position: sitting in a chair with a back rest with their feet flat on the floor and their arms hanging alongside their body. The evaluator placed the CROM device on the subject's head like a pair of eyeglasses and adjusted it with the strap. The evaluator then located the spinous process of $\mathrm{C} 7$ and placed the vertebra locator on it, adjusting the pressure until the subject indicated that he or she felt the pressure of the device. Once the subject felt pressure over C7, the evaluator stated, "From this moment you should not move." This procedure allowed the subject to become familiar with the test.

Next, the subject was asked to stand up and then sit back down again in the starting position. The evaluator, standing to the left of the subject, found the spinous process of $\mathrm{C} 7$ and placed the vertebra locator such that it formed a $90^{\circ}$ angle with the head arm of the CROM with the bubble indicating that the instrument was level (Figure 2A). This measurement was conducted twice; between the two measurements, the subject was asked to stand up and then sit back down again in the starting position.

\section{SMD}

For each subject, the evaluator first explained that the measurement would take place while lying on a couch. Also, at this time, the evaluator showed the digital caliper to the subject and said "You will notice contact on your sternum and on your chin; do not move." When the subject understood the statement and gave the evaluator permission to proceed, the subject was asked to lie in a supine position on the couch looking at the ceiling. When the subject was in position, the evaluator said "Don't move your head." Then, the distance from the jugular notch of the sternum to the protuberance of the chin was measured twice (Figure 2B); between the measurements, the subject was instructed to roll into a right lateral position and then return to the supine position.

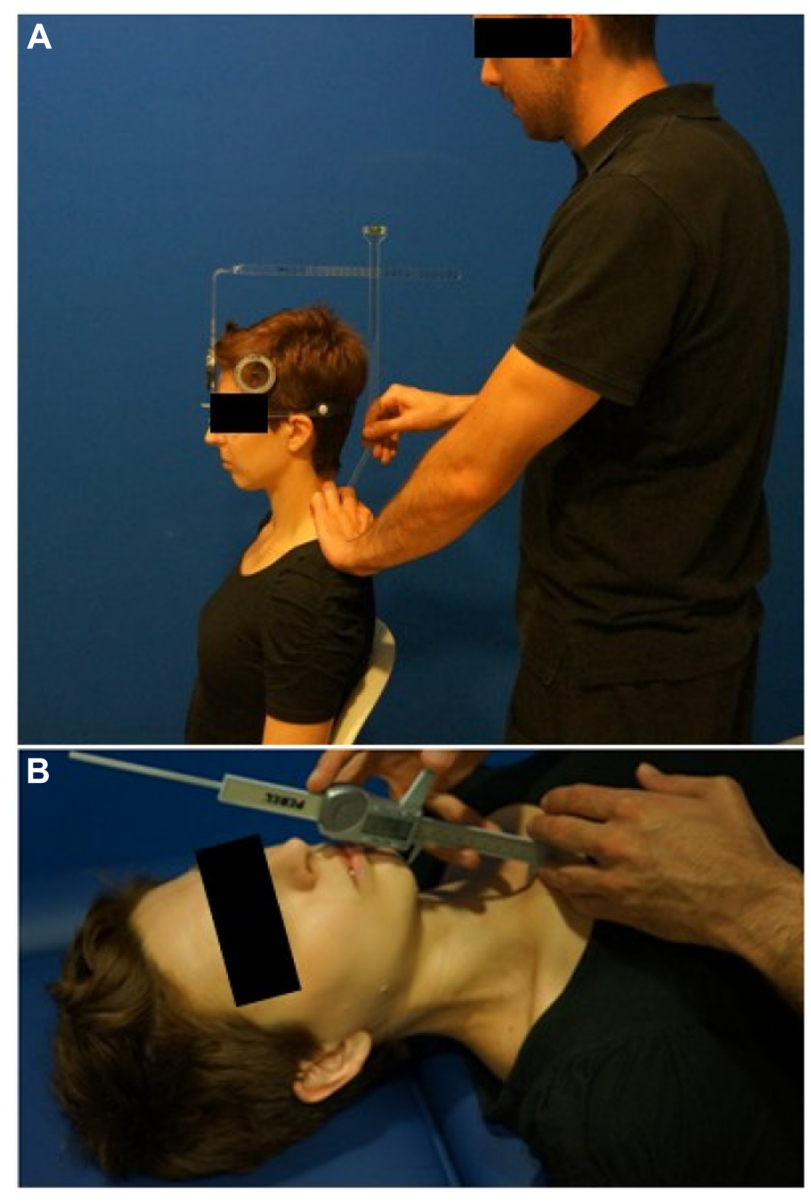

Figure 2 Craniocervical posture measurements.

Notes: (A) Placement of cervical range of motion device with the head arm for the measure of head posture with the vertebra locator. (B) Evaluator taking measure from the jugular notch of the sternum to the chin protuberance to quantitative the sternomental distance.

\section{Self-reported measures NDI}

The Spanish version of the NDI measures perceived neck disability. ${ }^{25}$ This questionnaire consists of ten items (seven questions related to daily living activities, two questions related to pain, and one question related to concentration) with each item scored from 0 (no disability) to 5 (complete disability) points. The NDI has demonstrated acceptable psychometric properties. $^{25}$

\section{CF-PDI}

The CF-PDI is a self-administered questionnaire designed to measure pain, disability, and functional status of the mandibular and craniofacial regions. ${ }^{23}$ This instrument consists of 21 items with a score ranging from 0 to 63 points. The CF-PDI has a good structure, internal consistency, reproducibility, and construct validity, and is an objective tool for assessing pain and disability in craniofacial pain patients. ${ }^{23}$ 


\section{Sample size}

The sample size was calculated using the method described by Walter et al, ${ }^{27}$ which is recommended for estimating sample sizes based on the intraclass correlation coefficient (ICC). A minimally acceptable ICC value of $P_{0}=0.7$ and an expected value of $P_{1}=0.8$ were chosen. To obtain a power of $80 \%(\beta=0.2)$ and a significance level of $5 \%$, we determined that a sample of at least 53 healthy subjects was required for intrarater reliability (two sets of two measurements were performed each day for 2 days). In addition, under the same conditions, we determined that a sample of at least 57 patients was required for intrarater reliability (one set of two measurements was performed each day for 3 days). To estimate the sample size, we used Power Analysis and Sample Size software (PASS 12).

\section{Data analysis}

The data were analyzed using the SPSS statistical package (SPSS v.20.0; IBM Corporation, Armonk, NY, USA). We used the Kolmogorov-Smirnov test to analyze the normal distribution $(P>0.05)$ of the variables. A value of $P<0.05$ was considered statistically significant.

The Pearson's correlation coefficient was used to analyze the association between HP and SMD in the two samples of subjects and was also used to analyze the correlations between the variables of pain-related disability with the HP and SMD data in patients with CCFP. A Pearson's correlation coefficient greater than 0.60 indicated a strong correlation, a coefficient between 0.30 and 0.60 indicated a moderate correlation, and a coefficient below 0.30 indicated a low or very low correlation..$^{28}$

We used the independent $t$-test to analyze the HP and SMD variables using the mean of trials 1 and 2 by comparing the collection data for the two samples. The effect sizes (Cohen's $d$ ) were calculated for the outcome variables. According to Cohen's method, the magnitude of the effect was classified as small $(0.20-0.49)$, medium $(0.50-0.79)$, or large $(\geq 0.8) .{ }^{29}$

The intrarater reliability was evaluated using the ICC. Reliability levels were defined based on the following classification: good reliability, ICC $\geq 0.75$; moderate reliability, ICC $<0.75$ and $\geq 0.50$; and poor reliability, ICC $<0.50 .^{30}$

Bland-Altman analysis was performed by calculating the mean difference between the two craniocervical posture measurements (HP and SMD) and the standard deviation (SD) of the difference. ${ }^{31}$ Ninety-five percent of the differences are expected to be less than two SDs. The closer the mean difference is to 0 and the smaller the SD of this difference, the better the agreement is. ${ }^{31}$
We expressed the measurement error as a standard error of measurement (SEM), which was calculated as:

$$
\mathrm{SD} \times \sqrt{1-\mathrm{ICC}}
$$

where SD is the SD of the values from all of the participants, and ICC is the reliability coefficient. ${ }^{32}$ The measurement error is the systematic and random error of a patient's score that is not attributable to the true changes in the construct being measured. ${ }^{33}$

Responsiveness was assessed using the minimal detectable change (MDC). $\mathrm{MDC}_{90}$ expresses the minimal change required to be $90 \%$ confident that the observed change between the two measures reflects real change and not measurement error; ${ }^{34}$ it is calculated as: ${ }^{34}$

$$
\mathrm{SEM} \times \sqrt{2} \times 1.96
$$

\section{Results}

The asymptomatic-subjects sample consisted of 53 participants, 30 of whom were women; the subjects ranged between 18 and 53 years of age. The symptomatic subjects included 60 CCFP patients, 32 of whom were women; the subjects ranged between 19 and 61 years of age. No statistically significant differences between the general characteristics of the two groups were found. The demographic characteristics data are summarized in Table 1. The group of symptomatic subjects presented a mean score of $14.78 \pm 4.04$ for neck disability and $16.30 \pm 7.11$ for craniofacial disability. All of the variables were normally distributed according to the Kolmogorov-Smirnov test. No subjects were excluded from the study based on the inclusion and exclusion criteria.

\section{Correlation study for chronic CCFP patients}

No statistically significant correlations between HP and SMD (measurements of craniocervical posture) and pain-related disability variables were detected (HP and neck disability $[r=0.105 ; P=0.43]$; HP and craniofacial disability $[r=0.132$; $P=0.31]$; SMD and neck disability $[r=0.126 ; P=0.33]$; SMD and craniofacial disability $[r=0.195 ; P=0.13]$ ).

The scatter diagram in Figure 3A shows a moderate positive correlation between HP and SMD $(r=0.56 ; P<0.001$; $\mathrm{n}=60$ ). Neck disability was strongly positively correlated with craniofacial disability $(r=0.79 ; P<0.001 ; \mathrm{n}=60)$.

In addition, the asymptomatic subject group showed a moderate positive correlation between HP and $\operatorname{SMD}(r=0.44$; $P=0.001 ; \mathrm{n}=53$; Figure 3B). 
Table I Demographic characteristics for the asymptomatic subjects' and chronic CCFP patients' samples

\begin{tabular}{llll}
\hline & $\begin{array}{l}\text { Asymptomatic } \\
\text { subjects }\end{array}$ & CCFP patients & $P$-value \\
\hline Age years & $38.1 \pm 10.5$ & $41.7 \pm 11.7$ & $0.099^{\#}$ \\
Sex (female) & $30(60)$ & $32(53.3)$ & $0.483^{\ddagger}$ \\
Height $(\mathrm{cm})$ & $169.6 \pm 8.1$ & $167 \pm 8.7$ & $0.102^{\#}$ \\
Weight $(\mathrm{kg})$ & $69 \pm 13.3$ & $71.2 \pm 10.5$ & $0.336^{\#}$ \\
\hline
\end{tabular}

Notes: Values are mean \pm standard deviation and $\mathrm{n}(\%)$. "Independent $t$-test. †Pearson's chi-squared.

Abbreviation: CCFP, cervico-craniofacial pain.

\section{Asymptomatic subjects versus chronic CCFP patients}

Independent $t$-tests were used to compare the asymptomatic and symptomatic groups with respect to the HP and SMD values using the mean values from trials 1 and 2 (separated by 48 hours). Statistically significant differences were found for both HP $(t=-3.89 ; P<0.001)$ and SMD $(t=-2.55 ; P=0.012)$. The effect sizes reached by these values were estimated to be small for SMD and medium for HP. The descriptive statistics, mean differences, and associated $95 \%$ confidence intervals (CIs) between the two samples are presented in Table 2 .

\section{Test-retest intrarater reliability for chronic CCFP patients}

The ICC value for intrarater reliability of single measures separated by 48 hours was 0.88 for HP and 0.79 for SMD. When the single measures were separated by 9 days, the ICC value for intrarater reliability was 0.81 for HP and 0.76 for SMD. The descriptive statistics, ICCs, and associated $95 \%$ CIs, SEMs, and $\mathrm{MDC}_{90}$ between trials are presented in Table 3.

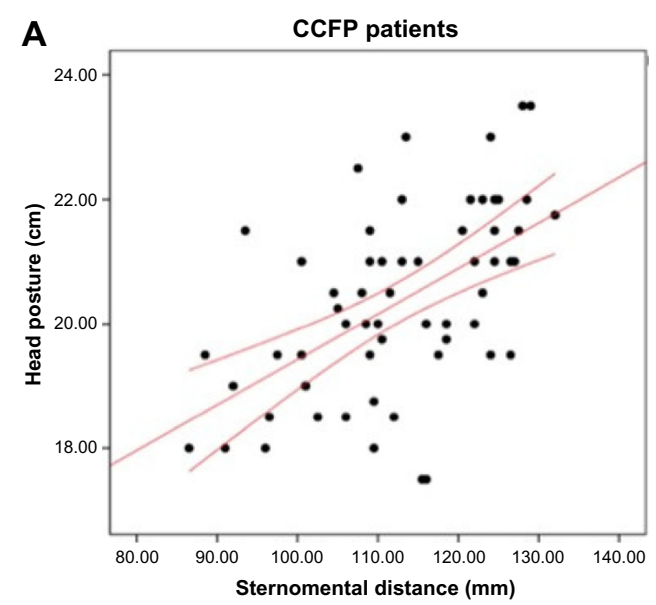

The Bland-Altman analysis for the intrarater performances for the assessment of HP and SMD is shown in Table 4. The mean differences in all of the Bland-Altman analyses were close to zero, suggesting appropriate intrarater and interrater reliability. The interrater performances of SMD at the $95 \%$ CIs showed large variability, indicating errors and suggesting that the SMD assessment is reliable but not precise (Table 4).

\section{Test-retest intrarater reliability for asymptomatic subjects}

The ICC value for intrarater reliability of single measures separated by 48 hours was 0.93 for HP and ranged from 0.95-0.99 for SMD. The descriptive statistics, ICCs, and associated $95 \%$ CIs, SEMs, and $\mathrm{MDC}_{90}$ between each evaluator's trials are presented in Table 3.

The Bland-Altman analysis for the intrarater performances is shown for the assessment of HP and SMD in Table 4. The mean differences in all of the Bland-Altman analyses were close to zero, suggesting appropriate intrarater reliability. The intrarater performances of SMD at the $95 \%$ CIs showed large variability, indicating errors and suggesting that the SMD assessment is reliable but not precise (Table 4).

\section{Discussion}

Regarding our main aim, we did not find an association between measurements of craniocervical posture and disability variables; this result is also supported by recent evidence. ${ }^{7,35,36}$ We note, however, that this issue is controversial. ${ }^{6,8}$ Previous studies have shown that craniofacial pain-related disability is associated with psychosocial factors and pain intensity. 23,37

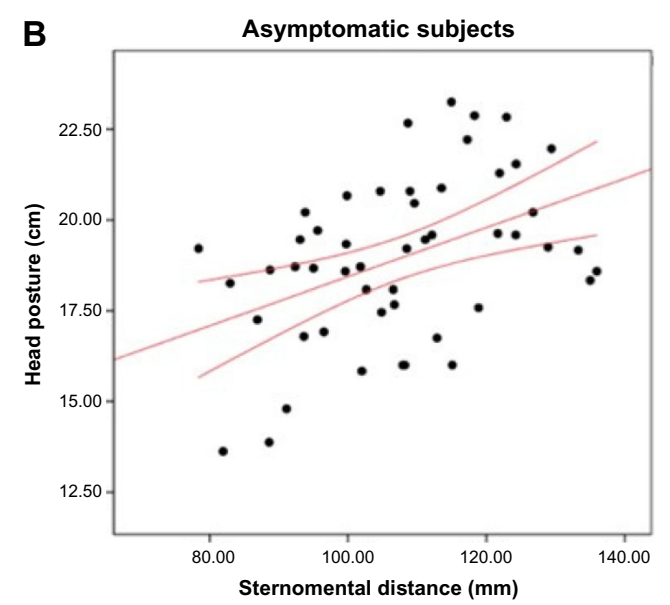

Figure 3 Scatter diagram showing correlation between head posture and the sternomental distance.

Notes: (A) Scatter diagram for chronic CCFP patients $(n=60)$. (B) Scatter diagram for asymptomatic subjects ( $n=53$ ). Graphic lines represent the $95 \%$ confidence interval. Abbreviation: CCFP, cervico-craniofacial pain. 
Table 2 Comparison between the asymptomatic subjects' and chronic CCFP patients' samples for measurements

\begin{tabular}{lllll}
\hline & $\begin{array}{l}\text { Asymptomatic subjects } \\
\text { (mean } \pm \text { SD) }\end{array}$ & $\begin{array}{l}\text { CCFP patients } \\
\text { (mean } \pm \text { SD) }\end{array}$ & $\begin{array}{l}\text { Mean differences } \\
(\mathbf{9 5 \%} \text { CI) }\end{array}$ & Cohen's d \\
\hline $\mathrm{HP}(\mathrm{cm})$ & $18.87 \pm 2.24$ & $20.32 \pm 1.49$ & $-1.44(-2.15 \text { to }-0.74)^{* *}$ & 0.76 \\
$\mathrm{SMD}(\mathrm{mm})$ & $106.31 \pm 14.25$ & $112.55 \pm 11.46$ & $-6.24(-11.1 \text { to }-1.38)^{*}$ & 0.38 \\
\hline
\end{tabular}

Notes: $* P<0.05 ; * * P<0.001$.

Abbreviations: CCFP, cervico-craniofacial pain; $\mathrm{Cl}$, confidence interval; HP, head posture; SD, standard deviation; SMD, sternomental distance.

According to our results, pain-related disability is not associated with craniocervical posture. However, we believe that craniocervical posture is a variable that should still be considered since previous scientific evidence has shown that it influences mandibular kinematics ${ }^{38-42}$ and pressure pain thresholds from craniofacial regions. ${ }^{38}$

We found a strong correlation between neck disability and craniofacial disability $(r=0.79)$; other studies have also reported similar results. ${ }^{23,35,36}$

Furthermore, the Pearson's correlation coefficient between $\mathrm{HP}$ and SMD is 0.447 for asymptomatic subjects and 0.56 for CCFP patients, suggesting a moderate correlation. We believe that this study is the first to demonstrate this association. Thus, we can believe that there is a relationship between HP in the sitting position and SMD in the supine position in healthy subjects.

Although we did find statistically significant differences between the healthy controls and the CCFP patients with respect to the mean HP and SMD values, with higher values in the CCFP group, the differences were very small, only slightly exceeding the MDC for the HP measurement (mean difference: $-1.44 \mathrm{~cm}$ ), and not exceeding the MDC for SMD (mean difference: $-6.24 \mathrm{~mm}$ ). Other studies have reported similar results, finding very small differences between the measurements of craniocervical posture in asymptomatic subjects versus symptomatic subjects with neck pain ${ }^{43}$ and TMD. ${ }^{35,36}$

HP should be considered when evaluating subjects in clinical practice. It can influence the pathophysiology of the cervical region. ${ }^{43,44}$ Our results show strong intrarater reliability when measuring HP with the CROM device. The results obtained with the digital caliper also reflected strong reliability for examining SMD. Several recent studies have measured HP using different methods and instruments. ${ }^{13-15,45}$ However, some of these studies were characterized by disadvantages such as low reliability, ${ }^{13,46}$ high cost, and difficulty in transporting the equipment. ${ }^{15,47,48}$ Furthermore, the risk of radiation exposure to the subject must be considered when a radiological diagnosis is used.

In the literature, we found only one study in which the intra- and interrater reliabilities in measuring HP using a
CROM device were evaluated. ${ }^{16}$ Similar to our results, this study showed good intra- and interrater reliabilities, although our interrater reliability was superior to that obtained by Garrett et $\mathrm{al}^{16}$ for asymptomatic subjects. Both investigations followed a rigorous standardized protocol using similar samples. An important aspect to note is that time did not influence the intrarater reliability; the results were very similar both 48 hours and 9 days later. It has been suggested that a range of 2-14 days between testing is generally acceptable for analyzing test reliability. ${ }^{49}$

As we noted earlier, SMD measured using the digital caliper showed high intrarater reliability and acceptable interrater reliability. Again, we found only one article in the literature that discussed SMD. However, that investigation was designed to generate a rule for predicting the degree of difficulty when performing a laryngoscopy. ${ }^{18,19}$ The mean SMD measurement in a study by Al Ramadhani et al ${ }^{18}$ was 142.8 ( $\mathrm{SD}=1.50$ ), whereas we found the SMD to range between 107.5 and 113.6 in our study. This difference could be explained by the fact that $\mathrm{Al}$ Ramadhani et al ${ }^{18}$ measured the cervical extension. It is also worth noting that their measurements were performed using a ruler with an accuracy of $5 \mathrm{~mm}$ rather than a digital caliper with a resolution of $0.01 \mathrm{~mm}$. We feel that this fact supports the contention that our investigation was more rigorous and reliable.

We found the intra- and interrater MDC of HP to vary from 1.27 to $1.74 \mathrm{~cm}$ and 0.96 to $1.30 \mathrm{~cm}$, respectively, and the intra- and interrater MDC of SMD varied from 3.76 to $14.55 \mathrm{~mm}$ and 16.13 to $16.47 \mathrm{~mm}$, respectively. The MDC is considered to be the smallest quantity above the SEM, although it should not be assumed that this change has reached the threshold of clinically significant improvement. ${ }^{50}$

\section{Clinical implications}

Our results show that measuring the craniocervical posture in asymptomatic subjects and patients has adequate reliability. This approach is clinically interesting because it is inexpensive and does not require much time.

Although no association was found between measurements of the craniocervical posture with disabilities variables, 

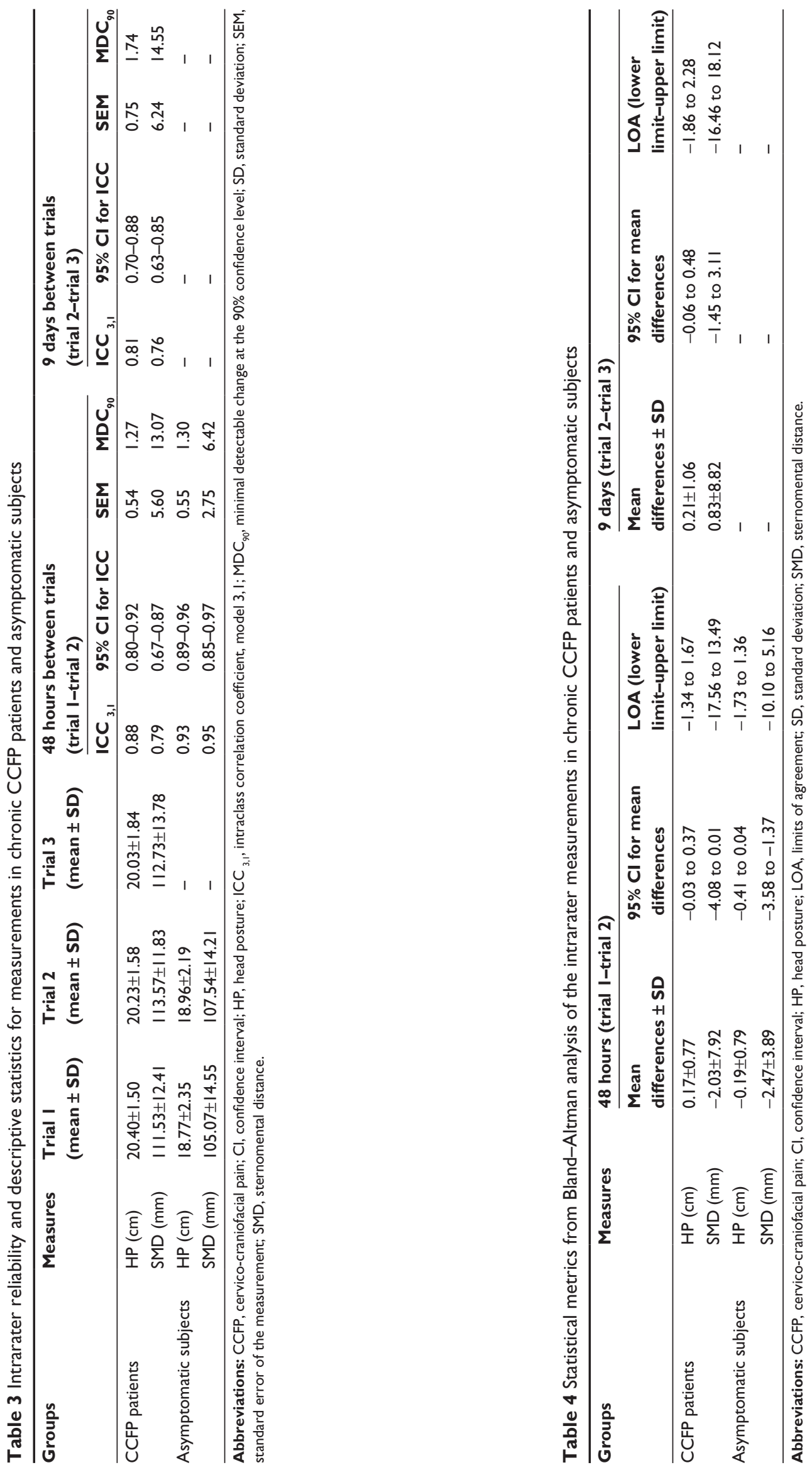
we believe that these measurements of craniocervical posture are important as a control measure for treatments based on therapeutic exercise. It is important to highlight that scientific evidence shows that craniocervical posture training with therapeutic exercise improves function and decreases pain in patients with craniofacial pain ${ }^{51}$ and CCFP. ${ }^{52,53}$

Our findings show a strong correlation between the two measures of pain-related disability. It has been suggested that it is important to assess disability in patients with craniofacial pain. ${ }^{54}$ In view of our results, we consider it essential to include pain-related disability measures in clinical evaluation of patients with CCFP.

\section{Limitations}

This study has several limitations that must be discussed. We agree with Garrett et $\mathrm{al}^{16}$ that a limitation exists in the head arm of the CROM in that it is marked in increments of 0.5 $\mathrm{cm}$, making it hard to determine a measurement when the indicator is between two marks. We believe that our reliability and data collection could be improved if the head arm was marked in millimeters. We also calculated the MDC but not the minimal clinically relevant change (MCRC), which we believe is of special interest in clinical practice. We must remember that the MDC is not the same as the MCRC, which is the grade of clinically significant improvement and is normally associated with an external criterion that indicates when change has occurred. ${ }^{50}$ We did not calculate the MCRC so we do not know the grade of clinically significant improvement. Future randomized controlled trials should identify interventions that influence HP and SMD, which could help assess the performance of this test when subjected to clinical interventions. Future results should also calculate the MCRC.

\section{Conclusion}

We did not find an association between measurements of craniocervical posture and disability variables. Furthermore, we noted a moderate correlation between HP and SMD and a strong correlation between neck disability and craniofacial disability. Our results suggest that there are small differences in the measures of the craniocervical posture between asymptomatic subjects and patients with CCFP. The CROM device and digital calipers are reliable instruments for measuring HP and SMD, respectively, in healthy subjects and CCFP patients. We also believe that additional studies should consider the MCRC and the influence of longer periods between examinations on the measures.

\section{Acknowledgment}

The study protocol was approved by the local ethics committee of the Center for Advanced Studies University La Salle, Madrid (Spain).

\section{Disclosure}

The authors report no conflicts of interest in this work.

\section{References}

1. Cheung Lau HM, Wing Chiu TT, Lam TH. Clinical measurement of craniovertebral angle by electronic head posture instrument: a test of reliability and validity. Man Ther. 2009;14:363-368.

2. Chaves TC, Turci AM, Pinheiro CF, Sousa LM, Grossi DB. Static body postural misalignment in individuals with temporomandibular disorders: a systematic review. Braz J Phys Ther. 2014;18:481-501.

3. Rocha CP, Croci CS, Caria PH. Is there relationship between temporomandibular disorders and head and cervical posture? A systematic review. J Oral Rehabil. 2013;40:875-881.

4. Haughie LJ, Fiebert IM, Roach KE. Relationship of forward head posture and cervical backward bending to neck pain. J Man Manip Ther. 1995;3:91-97.

5. Hanten WP, Olson SL, Russell JL, Lucio RM, Campbell AH. Total head excursion and resting head posture: normal and patient comparisons. Arch Phys Med Rehabil. 2000;81:62-66.

6. Yip CH, Chiu TT, Poon AT. The relationship between head posture and severity and disability of patients with neck pain. Man Ther. 2008; 13:148-154.

7. Cheung CH, Shum ST, Tang SF, Yau PC, Chiu TT. The correlation between craniovertebral angle, backpack weights, and disability due to neck pain in adolescents. J Back Musculoskelet Rehabil. 2010;23:129-136.

8. Lau KT, Cheung KY, Chan KB, Chan MH, Lo KY, Chiu TT. Relationships between sagittal postures of thoracic and cervical spine, presence of neck pain, neck pain severity and disability. Man Ther. 2010;15:457-462.

9. Fernández-de-Las-Peñas C, Cuadrado ML, Pareja JA. Myofascial trigger points, neck mobility and forward head posture in unilateral migraine. Cephalalgia. 2006;26:1061-1070.

10. Cimbiz A, Beydemir F, Manisaligil U. Evaluation of trigger points in young subjects. J Musculoskelet Pain. 2006;14:27-35.

11. Braun BL. Postural differences between asymptomatic men and women and craniofacial pain patients. Arch Phys Med Rehabil. 1991; 72:653-656.

12. Griegel-Morris P, Larson K, Mueller-Klaus K, Oatis CA. Incidence of common postural abnormalities in the cervical, shoulder, and thoracic regions and their association with pain in two age groups of healthy subjects. Phys Ther. 1992;72:425-431.

13. Silva AG, Punt TD, Johnson MI. Reliability and validity of head posture assessment by observation and a four-category scale. Man Ther. 2010;15:490-495.

14. Horton SJ, Johnson GM, Skinner MA. Changes in head and neck posture using an office chair with and without lumbar roll support. Spine (Phila Pa 1976). 2010;35:E542-E548.

15. Gadotti IC, Magee D. Assessment of intrasubject reliability of radiographic craniocervical posture of asymptomatic female subjects. J Manipulative Physiol Ther. 2013;36:27-32.

16. Garrett TR, Youdas JW, Madson TJ. Reliability of measuring forward head posture in a clinical setting. J Orthop Sports Phys Ther. 1993;17: 155-160.

17. Savva D. Prediction of difficult tracheal intubation. Br J Anaesth. 1994;73:149-153.

18. Al Ramadhani S, Mohamed LA, Rocke DA, Gouws E. Sternomental distance as the sole predictor of difficult laryngoscopy in obstetric anaesthesia. Br J Anaesth. 1996;77:312-316. 
19. Khan ZH, Mohammadi M, Rasouli MR, Farrokhnia F, Khan RH. The diagnostic value of the upper lip bite test combined with sternomental distance, thyromental distance, and interincisor distance for prediction of easy laryngoscopy and intubation: a prospective study. Anesth Analg. 2009;109:822-824.

20. von Elm E, Altman DG, Egger M, et al. The Strengthening the Reporting of Observational Studies in Epidemiology (STROBE) statement: guidelines for reporting observational studies. $J$ Clin Epidemiol. 2008;61:344-349.

21. Kottner J, Audige L, Brorson S, et al. Guidelines for Reporting Reliability and Agreement Studies (GRRAS) were proposed. Int J Nurs Stud. 2011;48:661-671.

22. La Touche R, París-Alemany A, Mannheimer JS, et al. Does mobilization of the upper cervical spine affect pain sensitivity and autonomic nervous system function in patients with cervico-craniofacial pain?: A randomizedcontrolled trial. Clin J Pain. 2013;29(3):205-215.

23. La Touche R, Pardo-Montero J, Gil-Martínez A, et al. Craniofacial pain and disability inventory (CF-PDI): development and psychometric validation of a new questionnaire. Pain Physician. 2014;17:95-108.

24. Dworkin SF, LeResche L. Research diagnostic criteria for temporomandibular disorders: review, criteria, examinations and specifications, critique. J Craniomandib Disord. 1992;6:301-355.

25. Andrade Ortega JA, Delgado Martínez AD, Almécija Ruiz R. Validation of the Spanish version of the Neck Disability Index. Spine (Phila Pa 1976). 2010;35:E114-E118.

26. Greenhalgh S, Selfe J. A qualitative investigation of Red Flags for serious spinal pathology. Physiotherapy. 2009;95:224-227.

27. Walter SD, Eliasziw M, Donner A. Sample size and optimal designs for reliability studies. Stat Med. 1998;17:101-110.

28. Hinkle D, Jurs S, Wiersma W. Applied Statistics for the Behavioral Sciences. 2nd ed. Boston: Houghton Mifflin; 1988.

29. Cohen J. Statistical Power Analysis for the Behavioral Sciences. Hillsdale: Lawrence Erlbaum Associates Inc.; 1988.

30. Portney LG, Watkins MP. Foundations of Clinical Research: Applications to Practice. New Jersey: Pearson/Prentice Hall USR; 2009.

31. Bland JM,Altman DG. Statistical methods for assessing agreement between two methods of clinical measurement. Lancet. 1986;1:307-310.

32. Weir JP. Quantifying test-retest reliability using the intraclass correlation coefficient and the SEM. J Strength Cond Res. 2005;19:231-240.

33. Mokkink LB, Terwee CB, Patrick DL, et al. The COSMIN study reached international consensus on taxonomy, terminology, and definitions of measurement properties for health-related patient-reported outcomes. $J$ Clin Epidemiol. 2010;63:737-745.

34. Haley SM, Fragala-Pinkham MA. Interpreting change scores of tests and measures used in physical therapy. Phys Ther. 2006;86:735-743.

35. Armijo-Olivo S, Rappoport K, Fuentes J, et al. Head and cervical posture in patients with temporomandibular disorders. J Orofac Pain. 2011;25:199-209.

36. Armijo-Olivo S, Magee D. Cervical musculoskeletal impairments and temporomandibular disorders. J Oral Maxillofac Res. 2013;3:e4.

37. Ozdemir-Karatas M, Peker K, Balık A, Uysal O, Tuncer EB. Identifying potential predictors of pain-related disability in Turkish patients with chronic temporomandibular disorder pain. $J$ Headache Pain. $2013 ; 14: 17$
38. La Touche R, París-Alemany A, von Piekartz H, Mannheimer JS, Fernández-Carnero J, Rocabado M. The influence of cranio-cervical posture on maximal mouth opening and pressure pain threshold in patients with myofascial temporomandibular pain disorders. Clin $J$ Pain. 2011;27:48-55.

39. Higbie EJ, Seidel-Cobb D, Taylor LF, Cummings GS. Effect of head position on vertical mandibular opening. J Orthop Sports Phys Ther. 1999;29:127-130

40. Isono S, Tanaka A, Tagaito Y, Ishikawa T, Nishino T. Influences of head positions and bite opening on collapsibility of the passive pharynx. $J$ Appl Physiol (1985). 2004;97:339-346.

41. Ohmure H, Miyawaki S, Nagata J, Ikeda K, Yamasaki K, Al-Kalaly A. Influence of forward head posture on condylar position. J Oral Rehabil. 2008;35:795-800.

42. Uritani D, Kawakami T, Inoue T, Kirita T. Characteristics of upper quadrant posture of young women with temporomandibular disorders. J Phys Ther Sci. 2014;26:1469-1472.

43. Silva AG, Punt TD, Sharples P, Vilas-Boas JP, Johnson MI. Head posture and neck pain of chronic nontraumatic origin: a comparison between patients and pain-free persons. Arch Phys Med Rehabil. 2009; 90:669-674.

44. Bonney RA, Corlett EN. Head posture and loading of the cervical spine. Appl Ergon. 2002;33:415-417.

45. Silva AG, Johnson MI. Does forward head posture affect postural control in human healthy volunteers? Gait Posture. 2013;38(2):352-353.

46. Juul-Kristensen B, Hansson GA, Fallentin N, Andersen JH, Ekdahl C. Assessment of work postures and movements using a video-based observation method and direct technical measurements. Appl Ergon. 2001;32:517-524.

47. Harrison DE, Haas JW, Cailliet R, Harrison DD, Holland B, Janik TJ. Concurrent validity of flexicurve instrument measurements: sagittal skin contour of the cervical spine compared with lateral cervical radiographic measurements. J Manipulative Physiol Ther. 2005;28:597-603.

48. van Niekerk SM, Louw Q, Vaughan C, Grimmer-Somers K, Schreve K. Photographic measurement of upper-body sitting posture of high school students: a reliability and validity study. BMC Musculoskelet Disord. 2008;9:113.

49. Streiner DL, Norman GR. Health Measurement Scales: a Guide to Their Development and Use. Oxford Oxford University Press; 1989.

50. Fletcher JP, Bandy WD. Intrarater reliability of CROM measurement of cervical spine active range of motion in persons with and without neck pain. J Orthop Sports Phys Ther. 2008;38:640-645.

51. Komiyama O, Kawara M, Arai M, Asano T, Kobayashi K. Posture correction as part of behavioural therapy in treatment of myofascial pain with limited opening. J Oral Rehabil. 1999;26:428-435.

52. Wright EF, Domenech MA, Fischer JR Jr. Usefulness of posture training for patients with temporomandibular disorders. J Am Dent Assoc. 2000;131:202-210.

53. Rota E, Evangelista A, Ciccone G, et al. Effectiveness of an educational and physical program in reducing accompanying symptoms in subjects with head and neck pain: a workplace controlled trial. J Headache Pain. 2011;12:339-345.

54. Ohrbach R. Disability assessment in temporomandibular disorders and masticatory system rehabilitation. J Oral Rehabil. 2010;37:452-480.

Journal of Pain Research

\section{Publish your work in this journal}

The Journal of Pain Research is an international, peer-reviewed, open access, online journal that welcomes laboratory and clinical findings in the fields of pain research and the prevention and management of pain. Original research, reviews, symposium reports, hypothesis formation and commentaries are all considered for publication.

The manuscript management system is completely online and includes a very quick and fair peer-review system, which is all easy to use. Visit http://www.dovepress.com/testimonials.php to read real quotes from published authors.

\section{Dovepress}

Submit your manuscript here: http://www.dovepress.com/journal-of-pain-research-journal 